\title{
A review on plastic bioaccumulation, potential health effects and the potential to enhance biotransformation using herbal medicine and nutritional supplements
}

\begin{abstract}
Background: Recent studies have highlighted that plastic contamination in air, food and drinking water is ubiquitous and evidence of plastic contamination in humans is growing. Plastic is a vector for heavy metal contamination and has been recently shown to cross the blood-brain barrier (BBB) in humans. Global concern regarding the health implications is mounting, with the World Health Organisation (WHO) having conducted a risk assessment review of plastics. Whilst knowledge gaps exist, we remain to eat, drink and inhale plastic toxins without knowing what harm it is potentially causing or how to detoxify it.
\end{abstract}

Aim: The aims of this review are to improve knowledge on the topic and to provide new insights on plastic bioaccumulation in humans, what is known about the potential health effects and how herbal medicine and nutritional supplements may assist plastic biotransformation.

Methods: I reviewed scientific articles in relation to the extent and sources of microplastic contamination, human biomonitoring studies, articles discussing potential human health implications, articles discussing how plastic toxins are biotransformed and articles examining herbal medicines and nutritional supplementation that support these detoxification processes.

Results: The results indicated biomonitoring studies in humans for persisted organic pollutants including toxins are not widespread, that research into biotransformation is a relatively new field and that no research exists that demonstrates how herbal medicines or nutritional supplements may enhance biotransformation to reduce the burden of human plastic contamination.

Conclusion: The findings help highlight that biomonitoring studies in humans need to be widely adopted to help ascertain the true extent of plastic bioaccumulation. Herbal medicine and nutritional supplements may be a valuable tool to enhance biotransformation of plastic contaminants in humans and further research into this area is warranted. The results and applications of this research have the potential to reduce endogenous EDCs and POPs in humans and thus may potentially lead to improved global health outcomes.

Keywords: plastic bioaccumulation, plastic biotransformation, microplastic, nanoplastic, microplastics in drinking-water, microplastic exposure and impacts on human health, complementary and alternative medicine (CAM), herbal medicine, nutritional supplements, pharmacognosy
Volume I 3 Issue I - 2020

\section{Carina Harkin}

Independent researcher, University College Cork (UCC), Ireland

Correspondence: Carina Harkin, Cert IVTAE, Independent researcher, Carahealth, Galway, University College Cork (UCC), Ireland,Tel 0834735746,Email carahealt@gmail.com

Received: January 14, 2020 | Published: January 20, 2020
Abbreviations: Acetyl-CoA; acetyl coenzyme A, ALS, amyotrophic lateral sclerosis; ADHD, attention deficit hyperactivity disorder; BPA, Bisphenol A; BBB, blood-brain barrier; CDG, Caclium D-Glucarate; CAT, catalase; CDC, centers for disease control and prevention; $\mathrm{CD}$, cluster of differentiation; $\mathrm{CoA}$, coenzyme A; CAM, complementary and alternative medicine; CuZn-SOD, copper-zinc SOD; CYP450, Cytochrome P450; DDT, dichlorodiphenyltrichloroethane; DINP, diisononyl phthalate; DIDP, Diisodecyl phthalate; EDCs, Endocrine disrupting chemicals; FSAI, Food Safety Authority of Ireland; Glutathione; GSH, glutathione peroxidase; GPx, GR, Glutathione reductase; GST, glutathione s-transferase; GST family, glutathione s-transferases; GMIT, galway- mayo institute of technology; GnRH, Gonadotropin-releasing hormone; HSE, Health Service Executive; HPA, hypothalamicpituitary axis; LGBTQI, lesbian, gay, bisexual, transgender, queer or questioning and intersex; bZIP, leucine zipper; Mn-SOD, Manganese SOD; MFO, mixed-function oxidase enzyme; NAC, N-acetylcysteine; NHANES, national health and nutrition examination survey; NATs, N-terminal acetyltransferases; NDDs, neurodevelopmental disorders; NDGs, neurodegenerative diseases; Nrf2, Nuclear factorerythroid-2-related factor 2; PCOS, polycystic ovary syndrome; POPs, Persistent organic pollutants; PTS, persistent toxic substances; PAHs, polycyclic aromatic hydrocarbons; PCBs, polychlorinated biphenyls; PETE, Polyethylene terephthalate; SAM-e, S-adenosyl- 
L-methionine; SFN, sulforaphane; SOD, superoxide dismutase; TcR, T-cell receptor; TCDD, tetrachlorodibenzo-p-dioxin; TEQ, toxic equivalent; T2D, type 2 diabetes; US, united states; UDP, uridine diphosphate; UDPGA, Uridine diphsphate glucuronic acid; UGT, UDP-glucuronosyltransferase; WHO, world health organisation

\section{Background}

\section{Plastic is a crude oil product a non-renewable energy source ${ }^{1}$}

The largest global consumer of oil is the United States (US) military, consuming 100million barrels of oil per year. ${ }^{2}$ Regardless of the ever-increasing abundance of evidence of fossil-fuelled climate change and billions in subsidies for alternative technologies, global oil consumption will reach 100million barrels per day, double what it was 50 years ago, with no sign of abating. ${ }^{3}$ The US alone uses 330 million barrels of oil per year in plastics production alone,${ }^{4}$ three times the US military use.

\section{The scale of microplastic contamination is staggering}

An estimated 8 million tons of plastic enters our oceans each year. Microplastics are omnipresent in sea waters, from deep-sea ocean sediments to the polar caps. ${ }^{5}$

\section{Microplastics contain and absorb toxic chemicals}

Microplastics are a vector for heavy metal contamination from the marine environment. ${ }^{6,7}$ Microplastics attract harmful pathogenic bacteria in sewage and contain and absorb toxic chemicals. More than 50 persistent organic pollutants (POPs), in particular polychlorinated biphenyls (PCBs) and polycyclic aromatic hydrocarbons (PAHs) are found in the five most common types of plastic. ${ }^{8}$ POPs are also called persistent toxic substances (PTS). ${ }^{9}$

\section{Plastic in tap water globally}

Billions of the global population are drinking water contaminated by plastic particles with $83 \%$ of samples found to be polluted. The US contamination rate was the highest at $94 \%$. The next highest rates were found in Lebanon and India. The lowest contamination rates at $72 \%$ were found in Germany, France and the UK. Each 500ml sample was found to contains on average 4.8 plastic fibres in the US and 1.9 plastic fibres in Europe. ${ }^{10,11}$

In 2017 an Irish study sampling tap and well water found microplastic contamination present in a small amount with the study author Dr Anne Marie Mahon, marine microplastic scientist from Galway-Mayo Institute of Technology (GMIT) advising that until we know what the health impact is we should adopt the precautionary principle. $^{12}$

\section{Plastic in bottled water globally}

The Orb study (Orb Media is a U.S-based non-profit journalism organisation) tested 259 bottles from 19 locations in 9 countries. 11 different brands were found to contain on average 325 plastic pieces of mircroplastic in every litre of water. Nile red dye was sued to fluoresce the particles of plastic in the water. This technique was developed by Dr Andrew Mayes of University of East Anglia. Nestlé Pure Life was found to have the highest levels of microplastic contamination with as many as 10,000 pieces of microplastic per litre. Only 17 of the 259 plastic water bottles tested were free of plastic. ${ }^{13}$ The contamination is thought to be in part derived from the packaging and bottling processes. ${ }^{14}$ Polypropylene plastic, the same plastic used to make bottle caps was the most common plastic piece found. Polypropylene (recycling number 5) is a considered a safe non-leaching plastic. The study was not published in a journal, nor has been peer reviewed. A second unrelated Story of Stuff Study in the US looked at 19 bottled water brands and found plastic pieces to be widely distributed. Bottled water was found to have twice the amount of microplastic than tap water (Appendix). ${ }^{15}$

\section{Appendix}

\section{The brands orb media tested}

a. Aqua (Danone)

b. Aquafina (PepsiCo)

c. Bisleri (Bisleri International)

d. Dasani (Coca-Cola)

e. Epura (PepsiCo)

f. Evian (Danone)

g. Gerolsteiner (Gerolsteiner Brunnen)

h. Minalba (Grupo Edson Queiroz), Nestlé Pure Life (Nestlé)

i. San Pellegrino (Nestlé)

j. Wahaha (Hangzhou Wahaha Group)

k. The bottles analysed were bought in the US, China, Brazil, India, Indonesia, Mexico, Lebanon, Kenya and Thailand.

\section{The brands story of stuff tested}

a. Boxed Water contained an average of 58.6 plastic fibres per litre.

b. Nestlé's Ozarka and Ice Mountain had concentrations at 15 and 11 pieces per litre, respectively.

c. Fiji Water had 12 plastic fibres per litre.

\section{Plastic water bottles}

Plastic water bottles are made from polyethylene terephthalate (PETE recycling code 1). This symbol is normally also found in soft drinks. PETE does not contain BPA or phthalates but studies have found that the endocrine disrupting chemical (EDC) antimony, a toxic phthalate 'plasticiser' used to make plastics flexible, leaches from PET bottles placed in the heat for prolonged periods of time. ${ }^{16}$ Reusable plastic drinking water bottles are or more generally now, were made from polycarbonate (recycling code 7). Polycarbonate is manufactured from BPA. ${ }^{17}$ Investigations show in some cases, BPAfree PETE containers might leach oestrogen-like chemicals. ${ }^{18}$

\section{Sources of environmental microplastic}

Washing acrylic, polyester and nylon: Plastic derived, acrylic, polyester and nylon persist in the environment. Synthetic clothingsetn to landfill breaks down to plastic microfiber pollution that contaminates ground and surface water. The estimates are that one truck load of clothes goes to landfill every second and one truckload of plastic enters the sea every second. Scientists estimate that plastic will outweigh fish in the sea by $2050 .{ }^{19}$ Acrylics are by far the most polluting with 
the average load of household washing shedding 750,000 microplastic fibres per load. This is 5 times greater than is shed by polyester-cotton. Drying synthetics in clothes dryer vents microplastic into the air. ${ }^{20,21}$

Burning plastics: Burring plastics releases endocrine disrupting cancer causing dioxins and furans the most toxic chemicals known to humankind. ${ }^{22}$

Human sludge: A 2017 study for the Environmental Protection Agency (EPA) co-written by Dr Anne Marie Mahon from the Marine from the Freshwater Research Centre, Galway-Mayo Institute of Technology (GMIT) identified sludge spreading and the washing of plastic by the recycling industry as significant sources of environmental microplastic. Estimates are that at least a billion microplastic fibres are spread on Irish farmland annually. ${ }^{23}$

Plastic in teabags: A recent study estimated that steeping one plastic containing teabag in $95{ }^{\circ} \mathrm{C}$ water temperature releases 11.6 billion microplastic fibres and 3.1 billion nanoplastic fibres into each cup of tea. The composition of particles released was found to be nylon and PETE matching the original teabags. Scientists conducted a toxicity assessment which showed that exposure to the plastic particles leaching from the teabags caused harmful behavioural and developmental health effects and that these were dose-dependent. ${ }^{15}$ Non-biodegradable plastic contain teabags have been added to the municipal compost for decades.

\section{Extent of plastic contamination in food and air}

Plastic in beer, salt and well water: German scientists tested 24 beer brands, honey and sugar and discovered microplastic fibres were found in all those tested. The study concluded the average person is ingesting 5,800 particles of plastic annually, with largest proportion $(88 \%)$ of this contamination being attributed to tap water consumption. ${ }^{10}$

Annual atmospheric microplastic fibres fallout on Paris: Microplastics and nanoplastic fibres in our sea evaporate to provide a source of both outdoor and indoor air pollution. A study examining microplastic atmospheric fallout estimated an annually fall-out of between 3 and 10 tons of microplastic on Paris. ${ }^{24}$

\section{Evidence of plastic contamination in humans}

Biomonitoring programmes: Biomonitoring human studies are evolving and useful for investigating exposure to phthalates, BPA and other chemicals. The U.S Center for Disease Control and Prevention (CDC) conducts a yearly National Health and Nutrition Examination Survey (NHANES). One of the objectives of the NHANEs is to assess the number of POPs detected in high concentrations in the population. NHANES results indicate it is common for people to have low and high concentrations of a number POPs. In relation to plastic POPs the NHANEs study measures;

a. Serum dioxins, furans and $\mathrm{PCB}$

b. Urinary phthalates and $\mathrm{BPA}^{25}$

One NHANEs study found 91 POPs analysed in blood samples and concluded that one tenth of the US population may have $\geq 10$ POPs each at concentrations in the top decile. ${ }^{26}$

\section{Plastic in human stools}

9 different plastics have been found in all human stools tested. ${ }^{27}$

\section{Latest cause for concern}

\section{Nanoplastic passes the BBB in fish}

Science has shown that nanoparticles or nanoplastic passes the blood-brain barrier (BBB) in fish resulting in behavioural disorders and brain damage. ${ }^{28}$

Most of the information we understand about the detrimental health effects of come from studies conducted in fish. The WHO is only just now looking into the health effects of plastics $n$ humans.

The WHO launched a health review on microplastics in drinking water to review the very scarce evidence which they described as scarce, to highlight evidence gaps, establish an agenda for research and allow for a more informed and thorough risk assessment.

\section{Endocrine disrupting chemicals and human health}

Endocrine Disrupting Chemicals (EDCs) include organochlorine pesticides, PCBs, BPA, phthalates, dioxins and furans..$^{29}$ Nearly 800 EDCs have the capacity to interfere with hormone receptors, hormone synthesis and hormone conversion. The three strands of evidence that give rise to concerns over EDCs include a high incidence and increasing rates of endocrine-related disease in the population, numerous observations of endocrine-related sequelae in the fauna and laboratory tests linking EDCs with detrimental human health outcomes. ${ }^{30}$

\section{EDC diseases and disorders}

The following information on EDC diseases and disorders is a summary of those highlighted in the State of the Science of Endocrine Disrupting Chemicals Report initiated by the WHO. ${ }^{30}$

Infertility in males and females: BPA can interfere with the hypothalamic-pituitary-ovarian/testicular axis (HPA), to increase hypothalamic gonadotropin-releasing hormone $(\mathrm{GnRH})$ secretion and promote pituitary proliferation. ${ }^{30,31}$

Low sperm count: Up to $40 \%$ of young males have a low sperm count. ${ }^{30}$

Genital malformations: Cryptorchidisms and hypospadias in baby boys. ${ }^{30}$

Sharp increase in children born with intersex variation IV: Ambiguous genitalia, hermaphrodite, pseudohermaphroditism etc.

Sharp increase in the incidence of gender dysphoria, transgender and gender neutral: It must be noted that Lesbian, Gay, Bisexual, Transgender, Queer or Questioning and Intersex (LGBTQI) existed pre-industrial revolution. ${ }^{30}$

Precocious puberty in young girls: This is a risk factor for breast cancer.

Adverse pregnancy outcomes: Including premature birth and low birth weight.

\section{Neurobehavioral disorders}

a. Cognitive, motor and sensory deficits.

b. Neurological impairments (NIs) including neuropathies.

c. Neurodevelopmental disorders (NDDs) including attention deficit hyperactivity disorder (ADHD) and autism. 
d. Neurodegenerative diseases (NDGs) including Parkinson's and Alzheimer's disease, and amyotrophic lateral sclerosis (ALS) ${ }^{30}$

e. POPs, BPA and phthalates exposure is associated. ${ }^{32}$

Hormone dependent tumours: Including breast, endometrial, ovarian, prostate, testicular and thyroid (30).

\section{Metabolic disorders:}

\section{Polycystic Ovary Syndrome (PCOS). ${ }^{30}$}

Metabolic syndrome: $:^{30}$ Evidence shows that EDCs may contribute to the evolution of the obesity pandemic and metabolic disorders including Type 2 Diabetes (T2D) ${ }^{33}$ Lipophilic POPs are linked to T2D. ${ }^{34}$

Atopic disorders: Phthalates and BPA exposure are linked to the pathogenesis of allergies, asthma and atopic dermatitis..$^{30,35}$

\section{Lowered vaccine response}

A Faroe Island study, where diets may include PCB contaminated whale blubber, on the vaccine repose of two birth cohorts, suggested that PCB exposure may reduce the immune response to childhood immunisations. ${ }^{36}$ Another study on the vaccine response of Dutch preschool children measured humoral immunity by antibody levels for mumps, measles, and rubella after primary vaccination and found that prenatal PCB exposure measured as a higher dioxin toxic equivalent (TEQ) was associated with an increased number of lymphocytes, T-cells, and cluster of differentiation (CD) CD8+ (cytotoxic), CD4+ (memory), T-cell receptor $(\mathrm{TcR})$ and $\mathrm{CD} 3(+)$ (activated) $\mathrm{T}$ cells and lower antibody levels to mumps and measles at preschool age. ${ }^{37}$

\section{Heath implications of microplastics according to the WHO}

It was recently widely reported that the WHO report referred to found no evidence of a current danger from microplastic. ${ }^{38}$ It must be noted that WHO report is about microplastic in drinking water only. Microplastic is as previously described universal. The WHO report in fact identified knowledge gaps and made recommendations in respect to monitoring and management of microplastic in the environment in order to better assess health risks posed to humans and to better inform appropriate management actions. ${ }^{39}$

\section{Microplastic mitigation}

BPA and phthalates and dioxin-like $P C B S$ are ingested plastic toxins.

Dioxins and furans are inhaled plastic toxins produced when plastic is burned. Dioxins, furans and dioxin-like PCBs are abbreviated names for a family of chemicals with similar toxicity and shared chemical characteristics. Dioxins and furans are also known as Tetrachlorodibenzo-p-dioxin (TCDD). TCDD was the contaminant in Agent Orange, the notorious herbicide used during the Vietnam War. ${ }^{40}$

\section{Mitigate environmental exposure}

Mitigation strategies include avoiding drinking water in plastic bottles and using an end stage water filtration unit to filter public drinking water supplies. Avoiding plastic incineration will also mitigate exposure. Phthalates are most typically found in industrial solvents and lubricants, additives in the textile industry, in pesticides, floorings, roofing, wall coverings, cables, clothing, packaging materials, personal-care products and toys. ${ }^{41} \mathrm{BPA}$ is used in the manufacture of epoxy and polycarbonate plastic resins. Products derived from BPA are commonly used in safety equipment, protective coatings inside tin cans and as composites and sealants in dentistry. BPA exposure primarily results from the ingestion of contaminated food. ${ }^{42}$

\section{Plastic biotransformation}

Measuring the burden of human exposure: BPA-glucuronide has terminal half-life of $<6 \mathrm{~h}$, is rapidly excreted in urine and can be used as a biomarker of exposure to BPA. Exposure to these phthalates can be assess using custom synthesised reference standards of specific oxidised metabolites of Diisononyl phthalate (DINP) and Diisodecyl phthalate $(D I D P)$. Phthalates are quickly metabolised and excreted in urine. ${ }^{43}$

\section{What happens after exposure?}

After ingestion, nanoplastic reaches the systemic blood circulation, distribute to a variety of body compartments and penetrates cells. ${ }^{44}$ Plastic too large to pass through the gut wall still present a risk to human health as it is a vector for hydrophobic POPs and EDCs of a smaller molecular size that are capable of penetrating cells. ${ }^{45}$

\section{Understanding biotransformation of plastic}

Research into human biotransformation and elimination systems is relatively new and continues to evolve.

\section{Phase I and II liver detoxification pathways}

Liver detoxification is a misnomer as Phase 1 and II Liver Detoxification Pathways also occur in kidney, intestine, lung, skin, prostate, and brain. In medical terminology toxicity is refered to as bioaccumulation and detoxification is referred to as biotransformation. Very little attention is being paid to human exposure to environmental chemicals and POPs.

Many toxic chemicals are fat-soluble, making them difficult to excrete. The P450 enzyme system turns lipophilic toxins into hydrophilic chemicals that are then able to be readuily excreted in urine, bile and sweat. In the event that fat lipophilic toxins are not made hydrophilic, lipophilic chemicals have a high affinity for fat tissues and cell membranes and can accumulate. These lipophilic toxins may be stored for years and released during times of exercise, stress or fasting. ${ }^{46}$

\section{Plastic biotransformation}

\section{Bisphenol A}

Accumulation of BPA induces cytochrome P450 (CYP450) enzyme activities. ${ }^{47}$ In humans BPA is mainly metabolised by $\mathrm{CYP} 2 \mathrm{C}$ and inhibits CYP17 activity ${ }^{48} \mathrm{BPA}$ is rapidly metabolised by glucuronidation conjugation. ${ }^{49} \mathrm{BPA}$ is also metabolite of sulphation and glutathione conjugation..$^{50}$ Transferases including sulfotransferases, glutathione- $S$-transferases (GSTs) are also involved in BPA conjugation. ${ }^{51} \mathrm{~A}$ fraction of absorbed BPA may distribute to body storage site (s) such as adipose tissue, followed by a slow, lowlevel release of BPA into the bloodstream. ${ }^{43}$

\section{Phthalates}

Phthalates undergo a series of phase I hydrolysis and phase II conjugation reactions and are subsequently excreted in faeces and urine. ${ }^{52}$ Phthalates induce CYP450 $0^{53}$ specifically CYP4 enzymes. ${ }^{54}$ 
Phthalates are a metabolite of glucuronidation, ${ }^{55,56}$ glycine and sulphation conjugation. ${ }^{56.57}$

\section{Dioxins, furans and dioxin-like PCBs}

People vary in capacity to eliminate TCDD. The elimination rate is much faster at higher than lower levels. ${ }^{58}$ TCDD induces a number of CYP450 enzymes systems..$^{59}$ Accumulation of POPs, dioxins ${ }^{59,60}$ and furans induce cytochrome P450 enzyme systems. ${ }^{61}$ Dioxins, furans and PCBs are metabolites of either glucuronidation or sulphate conjugation, mainly in the liver and excreted in the bile or urine. ${ }^{62}$ Dioxins undergo glutathione ${ }^{63}$ sulphation, ${ }^{64}$ glucuronidation ${ }^{40}$ and glycine conjugation phase II reactions. ${ }^{65}$

\section{Phase I - modification}

Phase I reactions occur in the liver and are catalysed by CYP450 mixed-function oxidase enzyme (MFO) chemical reactions including oxidation, reduction, hydrolysis, cyclization, decyclization (cyclization and decyclization have no relevance in plastic biotransformation) and hydroxylation. MFO enzymes inhabit hepatocytes membranes.

\section{Modification pathways involve}

a. Oxidation is process of being oxidised (combining chemically with oxygen/rust).

b. Reduction involves gaining of electrons by one atom involved in reaction.

c. Hydrolysis involves a chemical breakdown of a compound due to a water reaction.

d. Hydroxylation involves introduction of a hydroxyl group $(-\mathrm{OH}){ }^{46}$

\section{Induction of phase I (over activity)}

Certain metabolites of Phase I reactions are readily excreted. Many Phase I products are not rapidly eliminated and undergo subsequent Phase II reactions. If not adequately supported or when excessive POPs exist, Phase I is induced producing high levels of damaging free radicals. In cases whereby these reactive molecules are not readily metabolised by Phase II conjugation this can result in damage to proteins, RNA, and DNA. Phase I can turn a nontoxic molecule into a toxic (mutagenic/carcinogenic) molecule hence contribute to early ageing and cancer pathogenesis. ${ }^{66,67}$

\section{Phase II - conjugation}

In the hepatocytes, toxic metabolites undergo biotransformation and are conjugated using glutathione (GSH), sulphate, glucuronic acid or glycine. These reactions are catalysed by a large group of broad-specificity transferases, the most important being glutathione S-transferases (GSTs). Phase II conjugation reactions transform a fat soluble toxin into water-soluble chemical that can be excreted in bile, urine and sweat.

\section{Major phase II detoxification conjugation pathways include; ${ }^{46}$}

Glutathione (GSH): Co-factor: Glutathione and endogenous antioxidants; Glutathione S-transferase (GST), superoxide dismutase (SOD), glutathione peroxidise (GPx), glutathione reductase (GR) and catalase (CAT) ${ }^{68}$ The glutathione $S$-transferases (GST family) are activated through cysteine, glutamic acid and glycine to make glutathione. BPA, ${ }^{50}$ dioxins, furans and PCBs undergo glutathione conjugation. ${ }^{63}$

Sulphation: Co-factor: 3'-phosphoadenosine-5'-phosphosulfate (endogenously synthesised) Sulphation renders a xenobiotic less active but sometimes activates xenobiotics. Sulphation is involved in detoxification and hormone regulation. BPA, phthalates, ${ }^{50,56,57}$ dioxins, furans ${ }^{62,64}$ and PCBs undergo sulphation conjugation.

Glycine conjugation: Co-factor: Glycine is catalysed by glycine $\mathrm{N}$-acyltransferase.

Glycine conjugation is an important metabolic pathway which allows the for maintenace of sufficient coenzyme A (CoA) levels. Phthalates,${ }^{56,57}$ dioxins, ${ }^{65}$ furans and PCBs undergo glycine conjugation.

Glucuronidation: Co-factor: Uridine diphosphate (UDP) UDPglucuronic acid Uridine diphsphate glucuronic acid (UDPGA). $\beta$ glucuronidase breaks the chemical bonds formed during the detoxification processes, thus allowing the recirculation of toxins. ${ }^{69}$ BPA, phthalates, ${ }^{49,55,56,70}$ dioxins, furans and $\mathrm{PCBs}^{40}$ undergo glucuronidation conjugation.

\section{Pharmacognostical objectives}

a. Inhibit CYP450

b. Induce conjugation

\section{Andrographis/ Chuan Xin Lian/King of the bitters Andrographis paniculata}

Contains the diterpenolactones andrographolide, 14-deoxy11-dehydroandrographolide, 14-deoxy-11- oxoandrographolide, 5-hydroxy-7,8,2',3'-tetramethoxyflavone, neoandrographolide, paniculide-A, paniculide-B and paniculide-C inhibit CYP450. ${ }^{71}$

\section{Bupleurum/Chai hu bupleurum falcatum}

Contains saikosaponins which inhibit CYP450, specifically CYP1A2, CYP2C9 and CYP3A4. The flavonoids and steroids, rutin, isoquercetrin, isorhamnetin, quercetin, $\beta$-sitosterol, $\alpha$-spinasterol, daucosterol and $\alpha$-spinasterol glucoside inhibit CYP450 specifically CYP3A4..$^{71,72}$

\section{Burdock/nui bang zi arctium lappa}

Contains lignans lappaol F, diarctigenin and arctigenin which inhibit CYP450.71

\section{Centella/Ji Xue Cao Centella asiatica}

Contains triterpenes madecassic acid, brahmic acid and asiatic acid which inhibit CYP450. ${ }^{71}$

\section{Eleutherococcus/ci wu jia eleutherococcus senticosus}

Contains glycans eleutherans A, B, C, D, E, F, and G and eleutheroside $\mathrm{C}$ which inhibit CYP450.

\section{Scut Baic/huang qin scutellaria baicalensis}

Contains iridoid glycosides baicalein, wogonin and oroxylin which inhibit CYP450. 


\section{Inhibit phase I and induce phase II}

\section{Milk thistle silybum marianum}

Contains flavonolignans silymarin, silybin A, silybin $\mathrm{B}$, isosilybin A, isosilybin B, silychristin, isosilychristin, silydianin, and one flavonoid taxifolin ${ }^{73}$ which significantly inhibit CYP450. ${ }^{74}$ Silymarin restores depleted GSH to assist in glutathione conjugation. ${ }^{75}$ Milk thistle is rich in phytochemicals that can modulate UDPglucuronosyltransferase (UGT) Phase II enzymes. ${ }^{76}$

\section{Globe artichoke/Yang ji cynara scolymus}

Contains the flavonoid luteolin whihc inhibits CYP450 specifically CYP 3A4 and CYP3A5. ${ }^{77}$ Contains the hydroxycinnamic acid cynarin which promotes glucuronidation conjugation. Artichoke is a formidable antioxidant thus inhibiting toxin-induced glutathione reserve depletion. ${ }^{78}$

\section{Barberry/fu niu berberis vulgaris}

Contains the benzylisoquinoline alkaloid berberine which inhibits CYP450. ${ }^{79}$ Berberine specifically increases endogenous antioxidants, glutathione peroxidase (GPx) and superoxide dismutase (SOD), both copper-zinc SOD (CuZn-SOD) and manganese SOD (Mn-SOD) to assist glutathione conjugation. ${ }^{80}$

\section{Turmeric /jiang huang curcuma longa}

Contains the diarylheptanoid curcumin which inhibits CYP450 whilst inducing Phase II. ${ }^{81,82}$ Curcumin assists glutathione conjugation by restoring toxin-induced depleted GSH reserves. ${ }^{83}$ Curcumin inducesnuclear factor-erythroid-2-related factor 2 ( $N r f 2)$, a leucine zipper (bZIP) protein that regulates expression of antioxidant proteins thereby protecting against oxidative damage triggered by injury and inflammation. ${ }^{84} \mathrm{Nrf} 2$ regulates Phase-II enzymes. Curcumin modulates Phase I and Phase II enzymes. ${ }^{85}$

\section{Supplements}

\section{$\mathbf{N}$-acetylcysteine}

$\mathrm{N}$-acetylcysteine $(N A C)$ is a precursor to $\mathrm{L}$-cysteine that results in GSH elevation biosynthesis. NAC increases GSH conjugation and is a powerful antioxidant/free radical scavenger. ${ }^{86}$

\section{Caclium D-Glucarate}

Caclium D-Glucarate (CDG) inhibits $\beta$-glucuronidase, therefore enhances glucuronidation conjugation. ${ }^{87} \mathrm{CDG}$ is in cruciferous vegetables or vegetables from the Brassicaceae family.

\section{Sulforaphane}

Sulforaphane (SFN), an isothiocyanate found in cruciferous vegetables inhibits CYP450 and induces Phase II. ${ }^{88}$

\section{Hesperidin}

Hesperidin, a flavanone found in citrus fruits (oranges, lemons, pomelo), inhibits CYP450 and induces Phase II. ${ }^{89}$

\section{Grapefruit}

Grapefruit contains the compound naringenin which inhibits CYP450 and induces Phase II. ${ }^{54}$

\section{Conclusion}

As the awareness of microplastic contamination grows, so too does global concern regarding the health implications. Whilst reproductive and developmental abnormalities linked to EDC exposures in fauna are documented, the human health effects associated with full exposure of the whole gamut of endocrine-disrupting chemicals in the environment is yet to be determined, with the WHO report identifying knowledge gaps. Whilst some in the medical fraternity dismiss detoxification as quackery, bioaccumulation exists and is measurable. Research on human biotransformation and elimination systems and pharmacognostical approaches that support biotransformation of POPs, more specifically PCBs, BPA, phthalates, dioxins and furans, is urgently needed. In addition, mitigation strategies should be widely encouraged. CAM therapists who specialises in bioaccumulation and biotransformation may have a valuable role to play in detoxifying harmful EDCs in human beings. Further research into this area is warranted and the results and applications of this research have the potential to reduce endogenous EDCs and POPs in humans and thus, have the potential to lead to improved global health outcomes.

\section{Declarations}

a. Ethics approval and consent to participate Not applicable

b. Consent for publication Not applicable

c. Competing interests None declared

d. Funding None declared

e. Authors' contributions Carina Harkin wrote read and approved the final manuscript.

f. Acknowledgements Not applicable

g. Availability of data and materials not applicable.

\section{Acknowledgments}

None.

\section{Conflicts of interest}

Author declares that there are no conflicts of interest.

\section{Finding}

None.

\section{References}

1. Jennings MD. Oil and war: we had the warning 30 years ago. Nature. 2003;423(6935):15.

2. https://www.acq.osd.mil/eie/OE/OE_index.html

3. Cooper A, Johnson C. Now near 100 million bpd, when will oil demand peak? 2018

4. UK P. Plastic Production. 2018.

5. Jambeck JR, Geyer R, Wilcox C, et al. Marine pollution. Plastic waste inputs from land into the ocean. Science. 2015;347(6223):768-771.

6. Brennecke D, Duarte B, Paiva F, et al. Microplastics as vector for heavy metal contamination from the marine environment. Estuarine, Coastal and Shelf Science. 2016;178:189-195. 
7. Munier B, Bendell LI. Macro and micro plastics sorb and desorb metals and act as a point source of trace metals to coastal ecosystems. PloS One. 2018;13(2):e0191759.

8. Van A, Rochman CM, Flores EM, et al. Persistent organic pollutants in plastic marine debris found on beaches in San Diego, California. Chemosphere. 2012;86(3):258-263.

9. Wong MH, Armour MA, Naidu R, et al. Persistent toxic substances: sources, fates and effects. Rev Environ Health. 2012;27(4):207-213.

10. Kosuth M, Mason SA, Wattenberg EV. Anthropogenic contamination of tap water, beer, and sea salt. PLoS One. 2018;13(4):e0194970.

11. Kosuth M, Mason SA, Tyree C, et al. Synthetic polymer contamination in Global drinking Water. ORB. 2017.

12. Mahon AM, Nash R, O’Connor I. Research 210: Scope, Fate, Risks and Impacts of Microplastic Pollution in Irish Freshwater Systems. EPA GMIT; 2014.

13. Tyree C, Morrison D. Microplastics Found in Global Bottled Water. Orb Media. 2019.

14. Mason SA, Welch VG, Neratko J. Synthetic Polymer Contamination in Bottled Water. Front Chem. 2018;6:407.

15. Hernandez LM, Xu EG, Larsson HCE, et al. Plastic Teabags Release Billions of Microparticles and Nanoparticles into Tea. Environ Sci Technol. 2019;53(21):12300-12310.

16. Westerhoff P, Prapaipong P, Shock E, et al. Antimony leaching from polyethylene terephthalate (PET) plastic used for bottled drinking water Water Res. 2008;42(3):55155-55156.

17. Le HH, Carlson EM, Chua JP, et al. Bisphenol A is released from polycarbonate drinking bottles and mimics the neurotoxic actions of estrogen in developing cerebellar neurons. Toxicol Lett 2008;176(2):149-156

18. Yang CZ, Yaniger SI, Jordan VC, et al. Most plastic products release estrogenic chemicals: a potential health problem that can be solved Environ Health Perspect. 2011;119(7):989-996.

19. Gallo F, Fossi C, Weber R, et al. Marine litter plastics and microplastics and their toxic chemicals components: the need for urgent preventive measures. Environ Sci Eur. 2018;30(1):13.

20. Carney Almroth BM, Åström L, Roslund S, et al. Quantifying shedding of synthetic fibers from textiles; a source of microplastics released into the environment. Environmental science and pollution research international. Environ Sci Pollut Res Int. 2018;25(2):1191-1199.

21. Napper I, Thompson R. Release of synthetic microplastic plastic fibres from domestic washing machines: Effects of fabric type and washing conditions. Mar Pollut Bull. 2016;112(1-2):39-45

22. Marinković N, Pasalic D, Ferenčak G, et al. Dioxins and Human Toxicity. Arh Hig Rada Toksikol. 2010;61(4):445-453.

23. Mahon AM, Nash R, Connor IO. Scope, Fate, Risks and Impacts of Microplastic Pollution in Irish Freshwater Systems. Marine and Freshwater Research Centre, Galway-Mayo Institute of Technology: Environmental Protection Agency 2017

24. Dris R, Gasperi J, Saad M, et al. Synthetic fibers in atmospheric fallout: A source of microplastics in the environment? Mar Pollut Bull. 2016;104(1-2):290-293.

25. Committee on toxicity of chemicals in food CPATEC. National health and nutrition examination survey (NHANES) - Introduction. 2010.

26. Pumarega J, Gasull M, Lee DH, et al. Number of Persistent Organic Pollutants Detected at High Concentrations in Blood Samples of the United States Population. PloS One. 2016;11(8):e0160432.
27. Threat to human health from environmental plastics. BMJ. 2017;358.

28. Mattsson K, Johnson EV, Malmendal A, et al. Brain damage and behavioural disorders in fish induced by plastic nanoparticles delivered through the food chain. Sci Rep. 2017;7(1):11452.

29. Hood E. Are EDCs blurring issues of gender? Environ Health Perspect. 2005;113(10):A670-A677.

30. Åke Bergman JJH, Susan Jobling, Karen A, et al. State of the Science of Endocrine Disrupting Chemicals 2012. Geneva; World Health Organization (WHO): 2012. p. 1-38

31. Huo X, Chen D, He Y, et al. Bisphenol-A and Female Infertility: A Possible Role of Gene-Environment Interactions. Int $J$ Environ Res Public Health. 2015;12(9):11101-11116.

32. Zeliger HI. Exposure to lipophilic chemicals as a cause of neurological impairments, neurodevelopmental disorders and neurodegenerative diseases. Interdiscip Toxicol. 2013;6(3):103-110.

33. Le Magueresse-Battistoni B, Labaronne E, Vidal H, et al. Endocrine disrupting chemicals in mixture and obesity, diabetes and related metabolic disorders. World J Biol Chem. 2017;8(2):108-119.

34. Zeliger HI. Lipophilic chemical exposure as a cause of type 2 diabetes (T2D). Rev Environ Health. 2013;28(1):9-20.

35. Robinson L, Miller R. The Impact of Bisphenol A and Phthalates on Allergy, Asthma, and Immune Function: a Review of Latest Findings. Curr Environ Health Rep. 2015;2(4):379-387.

36. Heilmann C, Grandjean P, Weihe P, et al. Reduced Antibody Responses to Vaccinations in Children Exposed to Polychlorinated Biphenyls. PLOS Med. 2006;3(8):e311.

37. Weisglas-Kuperus N, Patandin S, Berbers GA, et al. Immunologic effects of background exposure to polychlorinated biphenyls and dioxins in Dutch preschool children. Environ Health Perspect. 2000;108(12):1203-1207.

38. Taylor AP. Microplastics in Drinking Water Don't Threaten Human Health. WHO. The Scientist Daily. 2019

39. WHO. Microplastics in drinking-water. Geneva: World Health Organization (WHO): 2019.

40. Bank PA, Salyers KL, Zile MH. Effect of tetrachlorodibenzo-p-dioxin (TCDD) on the glucuronidation of retinoic acid in the rat. Biochim Biophys Acta. 1989;993(1):1-6.

41. David RH, McKee RH, Butala JH, et al. Esters of Aromatic Mono, Di-, and Tricarboxylic Acids, Aromatic Diacids and Di-, Tri-, Or Polyalcohols. Wiley Online Library. 2001.

42. Kang JH, Kondo F, Katayama Y. Human exposure to bisphenol A. Toxicology. 2006;226(2-3):79-89.

43. Koch HM, Calafat AM. Human body burdens of chemicals used in plastic manufacture. Philos Trans $R$ Soc Lond B Biol Sci. 2009;364(1526):2063-2078.

44. Revel M, châtel A, Mouneyrac C. Micro(nano)plastics: A threat to human health? Current Opinion in Environmental Science \& Health. 2018;1:17-23.

45. Teuten EL, Saquing JM, Knappe DRU, et al. Transport and release of chemicals from plastics to the environment and to wildlife. Philos Trans R Soc Lond B Biol Sci. 2009;364(1526):2027-2045.

46. Drug metabolism. Wikipedia. 2018.

47. Takeshita A, Koibuchi N, Oka J, et al. Bisphenol-A, an environmental estrogen, activates the human orphan nuclear receptor, steroid and xenobiotic receptor-mediated transcription. Eur $J$ Endocrinol. 2001;145(4):513-517. 
48. Niwa T, Fujimoto M, Kishimoto K, et al. Metabolism and interaction of bisphenol $\mathrm{A}$ in human hepatic cytochrome P450 and steroidogenic CYP17. Biol Pharm Bull. 2001;24(9):1064-1067.

49. Ginsberg G, Rice DC. Does rapid metabolism ensure negligible risk from bisphenol A? Environ Health Perspect. 2009;117(11):1639-1643.

50. Jaeg JP, Perdu E, Dolo L, et al. Characterization of new bisphenol a metabolites produced by CD1 mice liver microsomes and S9 fractions. $J$ Agric Food Chem. 2004;52(15):4935-4942.

51. Shmarakov IO, Borschovetska VL, Blaner WS. Hepatic Detoxification of Bisphenol A is Retinoid-Dependent. Toxicol Sci. 2017;157(1):141155.

52. Frederiksen H, Skakkebaek NE, Andersson AM. Metabolism of phthalates in humans. Mol Nutr Food Res. 2007;51(7):899-911.

53. Takeshita A, Igarashi-Migitaka J, Nishiyama K, et al. Acetyl Tributyl Citrate, the Most Widely Used Phthalate Substitute Plasticizer, Induces Cytochrome P450 3A through Steroid and Xenobiotic Receptor. Toxicol Sci. 2011;123(2):460-470.

54. Hodges RE, Minich DM. Modulation of Metabolic Detoxification Pathways Using Foods and Food-Derived Components: A Scientific Review with Clinical Application. J Nutr Metab. 2015;2015:760689.

55. Stein TP, Schluter MD, Steer RA, et al. Autism and phthalate metabolite glucuronidation. J Autism Dev Disord. 2013;43(11):2677-2685.

56. Walter J, Crinnion JEP. Clinical Environmental Medicine. Identification and Natural Treatment of Diseases Caused by Common Pollutants. Elsevier; 2018. p. 520.

57. Phthalates and Cumulative Risk Assessment: The Tasks Ahead. National Academies Press. 2008.

58. Marinkovic N, Pasalic D, Ferencak G, et al. Dioxins and human toxicity. Arh Hig Rada Toksikol. 2010;61(4):445-453.

59. Schrenk D. Impact of dioxin-type induction of drug-metabolizing enzymes on the metabolism of endo- and xenobiotics. Biochem Pharmacol. 1998;55(8):1155-1162.

60. Kubota A, Watanabe MX, Kim EY, et al. Accumulation of dioxins and induction of cytochrome P450 1A4/1A5 enzyme activities in common cormorants from Lake Biwa, Japan: temporal trends and validation of national regulation on dioxins emission. Environ Pollut. 2012;168:131137.

61. Harrad S. Persistent Organic Pollutants: Environmental Behaviour and Pathways of Human Exposure. Harrad S, editor. West Sussex, United Kingdom: Chichester; 2001.

62. Daidoji T, Gozu K, Iwano H, et al. UDP-glucuronosyltransferase isoforms catalyzing glucuronidation of hydroxy-polychlorinated biphenyls in rat. Drug Metab Dispos. 2005;33(10):1466-1476.

63. Odell GB, Mogilevsky WS, Smith PB, et al. Identification of glutathione conjugates of the dimethyl ester of bilirubin in the bile of Gunn rats. Mol Pharmacol. 1991;40(4):597-605.

64. Dhakal K, He X, Lehmler HJ, et al. Identification of sulfated metabolites of 4-chlorobiphenyl (PCB3) in the serum and urine of male rats. Chem Res Toxicol. 2012;25(12):2796-2804.

65. Tarloff J.B. GRS, Hook J.B. Xenobiotic Metabolism in the Mammalian Kidney. Bach PH. LEA, editor. Dordrecht; Springer; 1987. p. 371-404.

66. Liska DJ. The detoxification enzyme systems. Altern Med Rev. 1998;3(3):187-198.

67. Omiecinski CJ, Vanden Heuvel JP, Perdew GH, et al. Xenobiotic metabolism, disposition, and regulation by receptors: from biochemical phenomenon to predictors of major toxicities. Toxicol Sci. 2011;120 Suppl 1(Suppl 1):S49-S75.

68. Pandey KB, Rizvi SI. Markers of Oxidative Stress in Erythro.cytes and Plasma During Aging in Humans. Oxid Med Cell Longev. 2010;3(1):212.

69. Badenhorst CP, van der Sluis R, Erasmus E, et al. Glycine conjugation: importance in metabolism, the role of glycine N-acyltransferase, and factors that influence interindividual variation. Expert Opin Drug Metab Toxicol. 2013;9(9):1139-1153.

70. Fay MJ, Nguyen MT, Snouwaert JN, et al. Xenobiotic Metabolism in Mice Lacking the UDP-Glucuronosyltransferase 2 Family. Drug Metab Dispos. 2015;43(12):1838-1846.

71. Mohamed L Ashour FSY, Haidy A Gad, Michael Wink2. Inhibition of Cytochrome P450 (CYP3A4) Activity by Extracts from 57 Plants Used in Traditional Chinese Medicine (TCM). Pharmacogn Mag. 2017;13(50):300-308.

72. Yu T, Chen X, Wang Y, et al. Modulatory effects of extracts of vinegarbaked Radix Bupleuri and saikosaponins on the activity of cytochrome P450 enzymes in vitro. Xenobiotica. 2014;44(10):861-867.

73. Polyak SJ, Morishima C, Lohmann V, et al. Identification of hepatoprotective flavonolignans from silymarin. Proc Natl Acad Sci U S A. 2010;107(13):5995-5999.

74. Kawaguchi-Suzuki M, Frye RF, Zhu H-J, et al. The effects of milk thistle (Silybum marianum) on human cytochrome P450 activity. Drug Metab Dispos. 2014;42(10):1611-1616.

75. Kim YC, Na JD, Kwon DY, Park JH. Silymarin prevents acetaminopheninduced hepatotoxicity via up-regulation of the glutathione conjugation capacity in mice. Journal of Functional Foods. 2018;49:235-240.

76. Mohamed ME, Frye R. Effects of Herbal Supplements on Drug Glucuronidation. Review of Clinical, Animal, and In Vitro Studies. Planta Med. 2010;77(4):311-321.

77. Quintieri L, Palatini P, Nassi A, et al. Flavonoids diosmetin and luteolin inhibit midazolam metabolism by human liver microsomes and recombinant CYP 3A4 and CYP3A5 enzymes. Biochem Pharmacol. 2008;75(6):1426-1437.

78. Gebhardt R. Antioxidative and protective properties of extracts from leaves of the artichoke (Cynara scolymus L.) against hydroperoxideinduced oxidative stress in cultured rat hepatocytes. Toxicol Appl Pharmacol. 1997;144(2):279-286.

79. Guo Y, Chen Y, Tan ZR, et al. Repeated administration of berberine inhibits cytochromes $\mathrm{P} 450$ in humans. Eur J Clin Pharmacol. 2012;68(2):213-217.

80. Lao-ong T, Chatuphonprasert W, Nemoto N, et al. Alteration of hepatic glutathione peroxidase and superoxide dismutase expression in streptozotocin-induced diabetic mice by berberine. Pharm Biol. 2012;50(8):1007-1012.

81. Muhammad I, Wang H, Sun X, et al. Dual Role of Dietary Curcumin Through Attenuating AFB(1)-Induced Oxidative Stress and Liver Injury via Modulating Liver Phase-I and Phase-II Enzymes Involved in AFB(1) Bioactivation and Detoxification. Front Pharmacol. 2018;9:554.

82. Krishnaswamy K, Goud VK, Sesikeran B, et al. Retardation of experimental tumorigenesis and reduction in DNA adducts by turmeric and curcumin. Nutr Cancer. 1998;30(2):163-166.

83. Jagatha B, Mythri RB, Vali S, et al. Curcumin treatment alleviates the effects of glutathione depletion in vitro and in vivo: therapeutic implications for Parkinson's disease explained via in silico studies. Free Radic Biol Med. 2008;44(5):907-917. 
84. NFE2L2 protein that regulates the expression of antioxidant proteins. Wikipedia. 2018.

85. Muhammad I, Wang H, Sun X, et al. Dual Role of Dietary Curcumin Through Attenuating AFB1-Induced Oxidative Stress and Liver Injury via Modulating Liver Phase-I and Phase-II Enzymes Involved in AFB1 Bioactivation and Detoxification. Front Pharmacol. 2018;9:554

86. Mokhtari V, Afsharian P, Shahhoseini M, et al. A Review on Various Uses of N-Acetyl Cysteine. Cell J. 2017;19(1):11-17.
87. Zoltaszek R, Kowalczyk P, Kowalczyk MC, et al. Dietary D-glucarate effects on the biomarkers of inflammation during early post-initiation stages of benzo[a]pyrene-induced lung tumorigenesis in $\mathrm{A} / \mathrm{J}$ mice. Oncol Lett. 2011;2(1):145-154.

88. Myzak MC, Dashwood RH. Chemoprotection by sulforaphane: keep one eye beyond Keap1. Cancer Lett. 2006;233(2):208-218.

89. Yahia EM. Fruit and Vegetable Phytochemicals: Chemistry and Human Health. Wiley Blackwell. 2017;2(25):1012. 